\title{
Machine Learning Approach for Data Analysis of Magnetic Orbital Moments and Magnetocrystalline Anisotropy in Transition-Metal Thin Films on $\mathrm{MgO}(001)$
}

\author{
KOHEI HAYASHI ${ }^{1}$ ABDUL-MUIZZ PRADIPTO,${ }^{1,4}$ KOHEI NOZAKI ${ }^{1}$ \\ TORU AKIYAMA, ${ }^{1}$ TOMONORI ITO,${ }^{1}$ TAMIO OGUCHI $, 2,3$ \\ and KOHJI NAKAMURA ${ }^{1,3}$
}

1.-Mie University, Tsu, Japan. 2.-Osaka University, Ibaraki, Japan. 3.-National Institute for Materials Science, Tsukuba, Japan. 4.—e-mail: a.m.t.pradipto@gmail.com

\begin{abstract}
Using the Least Absolute Shrinkage and Selection Operator (LASSO) technique, we analyze a long-standing issue in the field of magnetism: the relationship between orbital magnetic moments and magnetocrystalline anisotropy (MCA) energy in transition-metal thin films. Our LASSO regression utilizes the data obtained from first principles calculations for single slabs with six atomic-layers of binary $\mathrm{Au}-\mathrm{Fe}, \mathrm{Au}-\mathrm{Co}$, and $\mathrm{Fe}-\mathrm{Co}$ films on $\mathrm{MgO}(001)$. In the case of Fe-Co thin films, we have successfully regressed the MCA energy against the anisotropy of orbital moments along the in-plane and the perpendicular plane directions, giving a linear behavior. For the $\mathrm{Au}-\mathrm{Fe}$ and Au-Co thin films, however, our data-driven analysis shows no relation between the MCA energy and the anisotropy of orbital moments.
\end{abstract}

Key words: First principles calculation, magnetocrystalline anisotropy, orbital magnetic moment, single slab model

\section{INTRODUCTION}

Magnetic random access memory is expected as a next generation memory device due to several advantages such as the non-volatility, high speed reading and writing process as well as low power consumption. For the practical realization of the device, it is necessary to develop the magnetic tunnel junction element which consists of insulating and magnetic metal thin films with strongly preferred perpendicular magnetization with respect to the film plane. In this respect, materials containing strong spin orbit coupling (SOC) can be expected to have perpendicular magnetocrystalline anisotropy (MCA). This has been confirmed by calculations, for instance, in some Au-Fe binary systems. ${ }^{1}$ In such systems, while the magnetic moments are carried out by the $\mathrm{Fe}$ atoms or other $3 \mathrm{~d}$ transition metal

(Received July 15, 2018; accepted November 13, 2018; published online November 21, 2018)
(TM) elements, the strong SOC is introduced by the non-magnetic $\mathrm{Au}$ or other 5d TM elements. However, it was shown theoretically that the MCA of thin films depends strongly not only on the choice of atomic elements, but also on the detail of the atomic layer alignment. ${ }^{2}$ Designing novel magnetic metal multilayer films with a strong perpendicular MCA is, therefore, a very demanding task. The difficulty is in part related to the fact that MCA is related to many non-trivial but competing factors such as the SOC strength and crystal field, in a way that the full understanding of the origin of MCA has been one of the greatest issues in the field of magnetism.

In an effort to understand the MCA, it was suggested by Bruno ${ }^{3}$ that there are some proportionalities between MCA energy and the anisotropy of orbital moments, which will be referred to here as the Bruno relation. However, it was later known that the application of such relation may not apply generally. ${ }^{4}$ In particular, in systems containing atoms with strong SOC such as the 5d TM elements, such relation should be considered with extra care. ${ }^{4}$ 
In the present work, using the Least Absolute Shrinkage and Selection Operator (LASSO) technique, we examine the MCA and the anisotropy of orbital moments obtained from first principles calculations of different binary ferromagnetic multilayers including $\mathrm{Fe}$, Co and $\mathrm{Au}$ atoms to analyze the Bruno relation. Our analysis indeed confirms that the relation is applicable for systems without $\mathrm{Au}$, i.e., the considered $5 \mathrm{~d}$ element. However, in the $\mathrm{Fe}-$ $\mathrm{Au}$ and $\mathrm{Co}-\mathrm{Au}$ systems, the relationship deviates strongly from the Bruno relation.

\section{MODEL AND METHOD}

Self-consistent Density Functional Theory calculations were performed based on the generalized gradient approximation $(\mathrm{GGA})^{5}$ in the scalar relativistic approximation (SRA), i.e., without SOC, by using the Full-potential Linearized Augmented Plane-Wave (FLAPW) method. ${ }^{6-8}$ In all calculations, two-dimensional film geometries with additional (non-periodic) vacuum regions have been considered. We model single slabs with six-atomic layers of binary Au-Fe, Au-Co and Co-Fe films on top of four layers of $\mathrm{MgO}$ (001) substrates. All atomic-layer configurations, which include as many as $2^{6}=64$ possibilities for each binary system, were considered in the calculations (see Fig. 1). A planewave cut-off $|\mathrm{k}+\mathrm{G}|$ of $3.9 \mathrm{a}$. $\mathrm{u}^{-1}$ has been chosen, and we choose suitable muffin-tin radii of $2.3 \mathrm{bohr}$ for $\mathrm{Au}$ and $2.2 \mathrm{bohr}$ for both Co and Fe.

The MCA energy, $E_{\mathrm{MCA}}$, is defined in our calculations as the energy eigenvalue difference between the magnetizations oriented along the in-plane [100] and perpendicular [001] directions with respect to the film plane. To determine the MCA energy, the second variational method ${ }^{1,9}$ for treating SOC by using the calculated eigenvectors in the SRA has been employed, and then the MCA energy has been calculated by using the force theorem. ${ }^{10-12}$ The number of special k-points in the two-dimensional Brillouin zone (BZ) was 8100, and it is found that such k-point mesh was large enough to suppress numerical fluctuations in the MCA energy by less than $0.01 \mathrm{meV}$, which is a sufficient accuracy for the purpose of the present work.

In regression analysis, we consider the linear regression model in which the MCA energy is expressed by using the orbital magnetic moment,

$$
\begin{aligned}
E_{\mathrm{MCA}}= & \sum_{i=1}^{6} A^{\mathrm{Au}, i} M_{\mathrm{anis}}^{\mathrm{Au}, i}+B^{\mathrm{Fe}, i} M_{\mathrm{anis}}^{\mathrm{Fe}, i}+C^{\mathrm{Au}, i} M_{\mathrm{ave}}^{\mathrm{Au}, i} \\
& +D^{\mathrm{Fe}, i} M_{\mathrm{ave}}^{\mathrm{Fe}, i} .
\end{aligned}
$$

Here, the MCA energy has been expanded by using the descriptors, which for the present work, we choose the anisotropies and averages of orbital moments. The orbital moment anisotropy $M_{\text {anis }}^{\mathrm{Au} / \mathrm{Fe}, i}$ is defined as the difference between the orbital moments of the perpendicular and in-plane
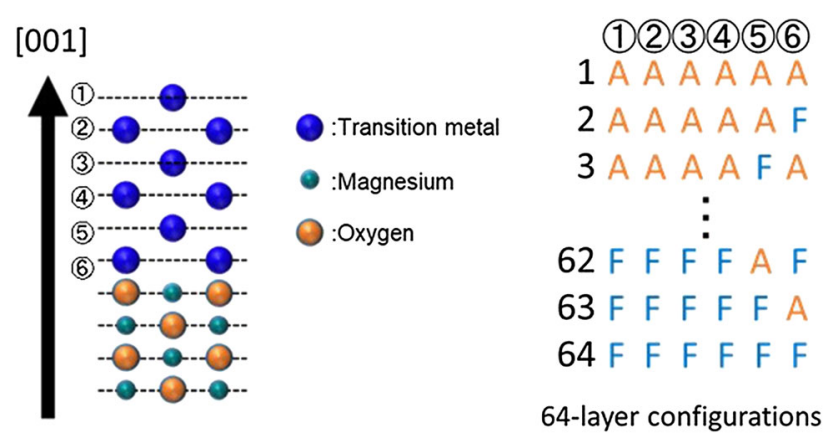

Fig. 1. Calculation model and notation for atomic layer configurations of $\mathrm{TM} / \mathrm{MgO}$, where $\mathrm{TM}$ is composed by $\mathrm{Au}, \mathrm{Fe}$ or Co. The perpendicular and in-plane directions are [001] and [100], respectively. All atomic layer patterns $\left(2^{6}=64\right)$ expressed by binary configurations are considered as illustrated in the right side of the figure.

magnetizations for $\mathrm{Au}$ or $\mathrm{Fe}$ species at the $i$ th layer. Likewise, the orbital moment average $M_{\mathrm{anis}}^{\mathrm{Au} / \mathrm{Fe}, i}$ is defined as the average of the perpendicular and inplane orbital moments of the respective atomic species. To eliminate the bias of choosing the descriptor, we consider that is not only the anisotropy, but also the average included. A leave-oneout cross validation, where the data is separated to 63 training data and one test data, was applied. Coefficients $A, B, C$ and $D$ in the Eq. 1 show how the descriptors are correlated to the parameters. For example, a large $A^{\text {Au,1 }}$ indicates that the anisotropy of orbital moment of the $\mathrm{Au}$ atomic layer placed at the top layer, i.e., layer 1 , strongly affects the prediction of $E_{\mathrm{MCA}}$. The $A, B, C$, and $D$ coefficients are determined by using the training data, and then used to predict $E_{\mathrm{MCA}}$ of the test data.

\section{RESULTS}

The calculated results of MCA energy and anisotropy of orbital moments as functions of the atomic composition for each binary system are shown in Fig. 2. Both the computed values diverge largely even within the same atomic composition. For a specific atomic composition, e.g., for $\mathrm{F}_{3} \mathrm{Co}_{3}$ in the Co-Fe binary system, the MCA energy varies largely from in-plane anisotropy with an $E_{\mathrm{MCA}}$ of about - $1.5 \mathrm{meV} /$ unit-area, to a perpendicular one with an $E_{\mathrm{MCA}}$ of about $1.5 \mathrm{meV} /$ unit-area. The variations are even larger in the cases of $\mathrm{Au}-\mathrm{Fe}$ and $\mathrm{Au}-\mathrm{Co}$ binary systems. This observation is consistent with previous theoretical work. ${ }^{1}$ A similar behavior is observed for the anisotropy of orbital moments. These results show that both MCA energy and anisotropy of orbital moments depend not only on the atomic composition, but more importantly, they depend also on the stacking sequence of these atoms.

In the Co-Fe binary systems, all atomic layers carry magnetic moments. Figure 3a shows a scatter plot between $E_{\mathrm{MCA}}$ and $M_{\text {anis }}$ which suggest a linear relationship, as visualized with the red line. Hence, 

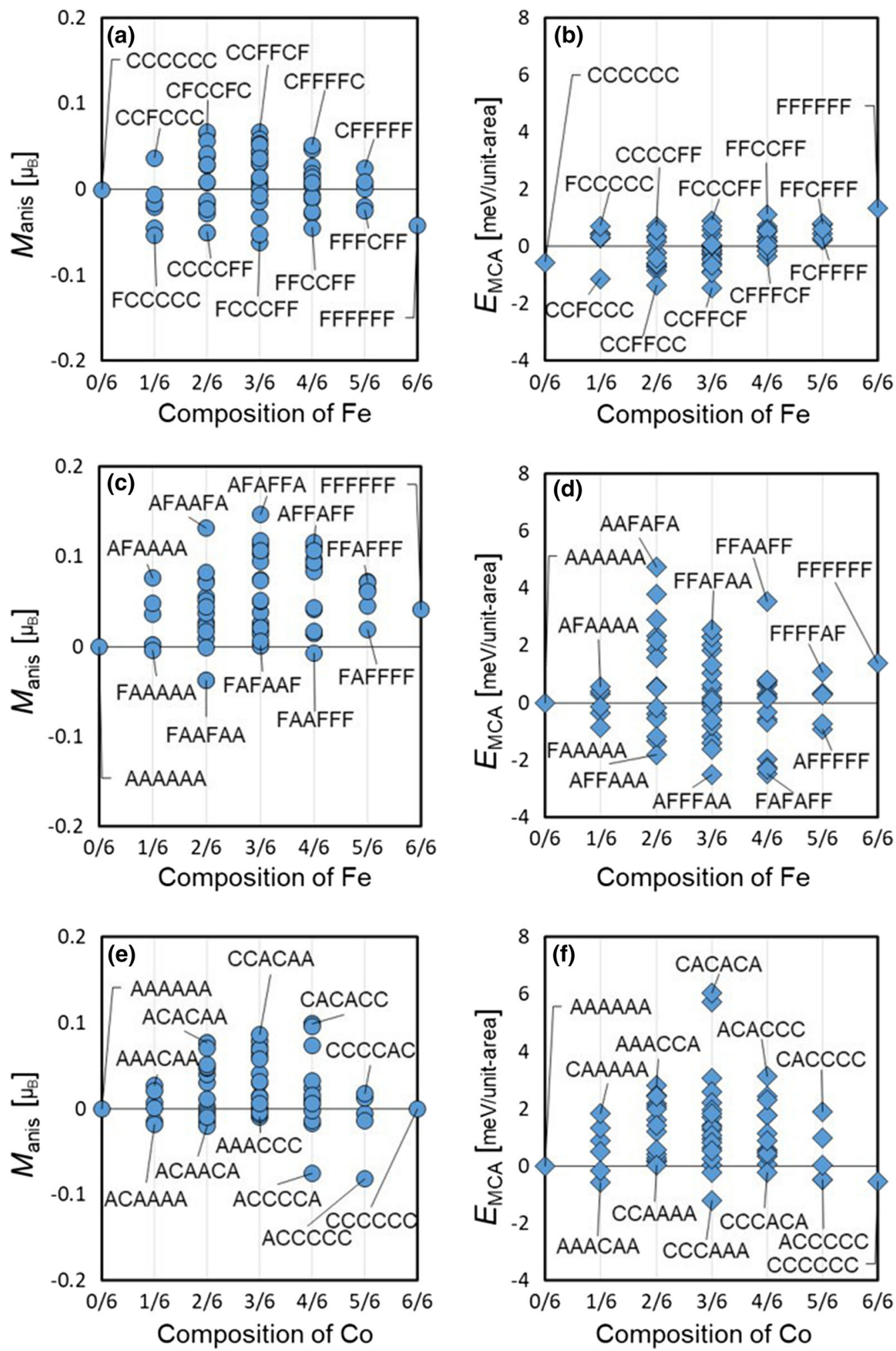

Fig. 2. Results of anisotropy of orbital moments, $M_{\text {anis }},(a, c, e)$ and MCA energies, $E_{\mathrm{MCA}}$, (b, d, f) as functions of the composition for Co-Fe, Au$\mathrm{Fe}$, and $\mathrm{Au}-\mathrm{Co}$ binary systems. Atomic layer configurations are represented, e.g., by CCFFCF for $\mathrm{CoCoFeFeCoFe} / \mathrm{MgO}$, where $\mathrm{C}$, $\mathrm{F}$, and $\mathrm{A}$ indicate $\mathrm{Co}, \mathrm{Fe}$, and $\mathrm{Au}$ atomic layers, respectively.

for this Co-Fe binary system, an agreement with Bruno relation ${ }^{3}$ is obtained. We further continue with LASSO analysis using the coefficients of the
$M_{\text {ave }}$ and $M_{\text {anis }}$ for each layer and atom species. The results are shown in Fig. 3b. From this figure, it is visible that the coefficients of almost all $M_{\text {anis }}$ show 


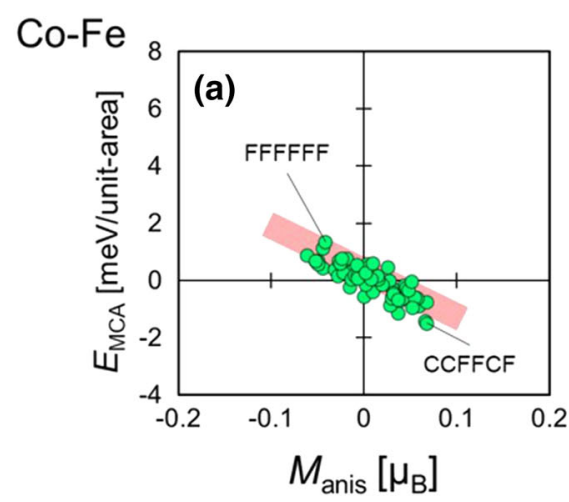

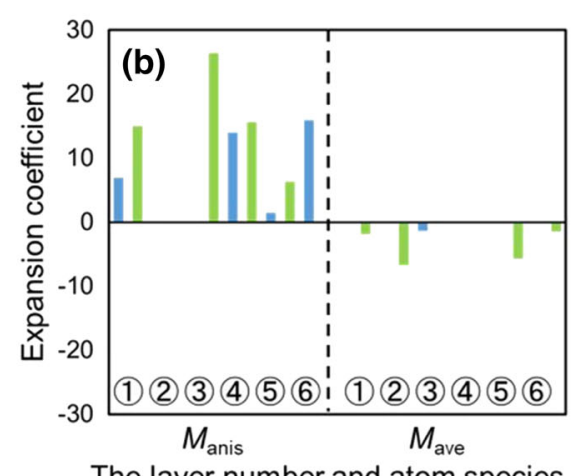

The layer number and atom species

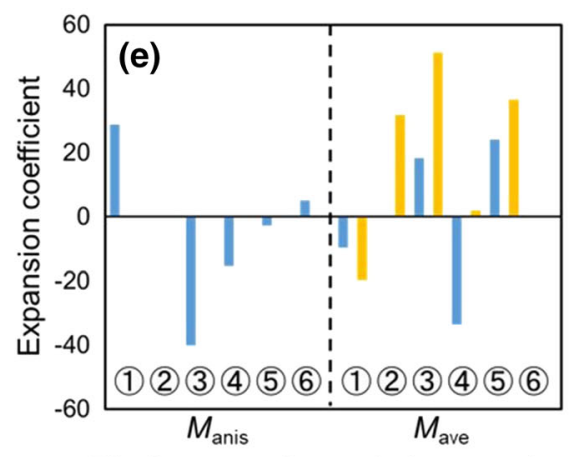

The layer number and atom species

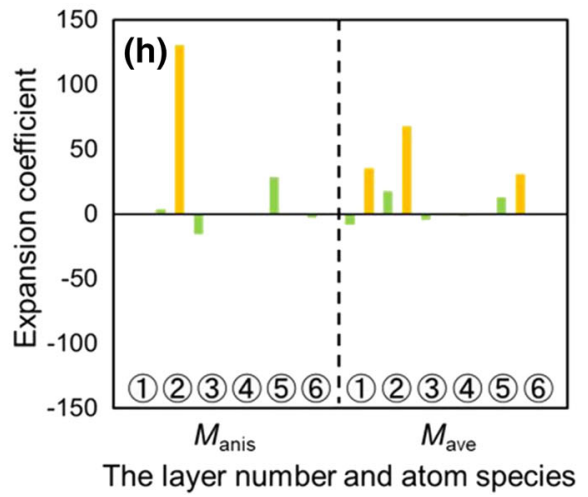

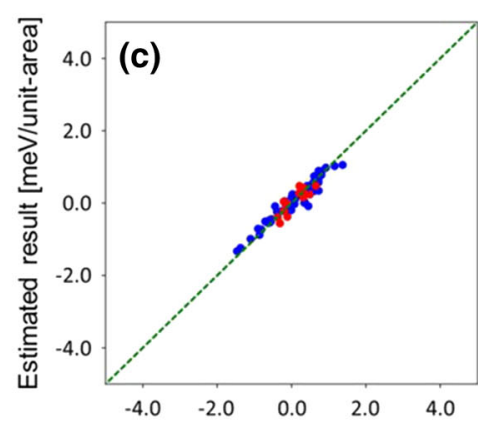

First principle result [meV/unit-area]
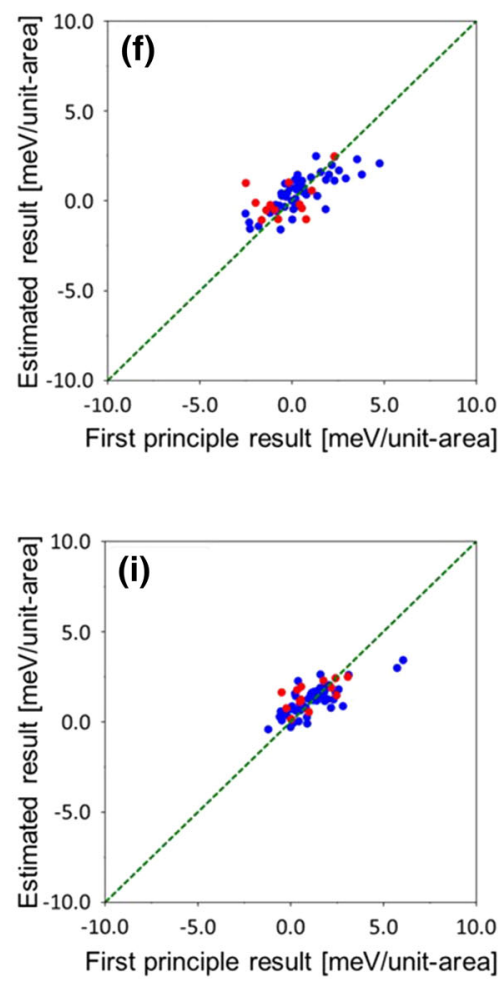

Fig. 3. (a, d, g) Plots between MCA energy, $E_{\mathrm{MCA}}$, and anisotropy of orbital moments, $M_{\text {anis }}$, for Co-Fe, Au-Fe, and Co-Fe binary systems. (b, e, h) Calculated coefficients for anisotropy and average values of orbital moments in Eq. 1, where $M_{\text {anis }}$ and $M_{\text {ave }}$ indicate, respectively, the anisotropies and averages of the orbital moment. The numbers in the horizontal axis denote the atomic layer positions in the film from the surface (1) to the interface (6) (c, f, i) Comparison of the MCA energies calculated by the first principle calculations and estimated by using the LASSO coefficients. Red and blue solid circles show the data of training and test.

large values, implying the significance of all anisotropy of orbital moments. Figure 3c further shows the comparison between the calculated $E_{\mathrm{MCA}}$ from first principles and the estimated ones using LASSO. These results show that the $E_{\mathrm{MCA}}$ values predicted using LASSO agree well those obtained explicitly by using first principles calculations.

In the $\mathrm{Au}-\mathrm{Fe}$ binary systems, the $E_{\mathrm{MCA}}$ varies largely from $4.8 \mathrm{meV} /$ unit-area with a perpendicular anisotropy to $-2.5 \mathrm{meV} / \mathrm{unit}$-area with an inplane one. Similarly, $M_{\text {anis }}$ varies from 0.15 to $-0.05 \mu_{\mathrm{B}}$. From Fig. 3d, no simple relation between $E_{\mathrm{MCA}}$ and $M_{\text {anis }}$ is visible. Figure $3 \mathrm{e}$ shows the results analyzed by LASSO for the Au-Fe binary systems. While there are few descriptors which are significant for the prediction of $E_{\mathrm{MCA}}$, a clear correlation is missing. This leads to the unsatisfactory prediction of the $E_{\mathrm{MCA}}$ calculated by DFT, as shown in Fig. 3f.

Finally, in the Au-Co binary systems, there is also a similarly large variation of $E_{\mathrm{MCA}}$ from $6.1 \mathrm{meV} /$ unit-area to $-1.2 \mathrm{meV} /$ unit-area, while the $M_{\text {anis }}$ varies from $0.12 \mu_{\mathrm{B}}$ to $-0.08 \mu_{\mathrm{B}}$. From LASSO analysis, as shown in Fig. 3h, there is also no simple correlation between $E_{\mathrm{MCA}}$ and $M_{\text {anis. }}$

In both binary systems, which include an $\mathrm{Au}$ element, the agreements between the $E_{\mathrm{MCA}} \mathrm{S}$ 
calculated by first principle calculations and those predicted using the LASSO coefficients are not satisfying. In the first place, the Bruno relation does not simply apply in these systems due to the strong SOC of Au, as suggested in a previous work. ${ }^{4}$ Indeed, the induced magnetic moments in Au due to the presence of magnetic TM-3d are from 0.0 to 0.25 $\mu \mathrm{B}$, suggesting a very small exchange splitting on the Au sites. Combined with a strong SOC, such small splitting will lead to important spin-flip term to the SOC energy, resulting in a breakdown of the simple Bruno relation. Additionally, in the present analysis, we employ only the orbital moment anisotropies and averages as the data descriptors for the MCA energy. The discrepancy between the first-principles calculated and the predicted $E_{\mathrm{MCA}}$ indicates that such descriptors might not be sufficient to predict the MCA energy of systems with strong SOC.

\section{CONCLUSIONS}

From our combined first-principles calculations and machine learning analysis, we show that the $E_{\mathrm{MCA}}$ of the Co-Fe thin films is strongly correlated to the anisotropy of orbital moments, and that the estimated $E_{\mathrm{MCA}}$ shows a good agreement with the calculated one. The $E_{\mathrm{MCA}} \mathrm{S}$ of the $\mathrm{Au}-\mathrm{Fe}$ and Co-Fe show only a weak correlation with the anisotropy of orbital moments.

\section{ACKNOWLEDGMENTS}

Work was supported by JSPS KAKENHI Grant Numbers 15H05702, 16K05415, and 17H06154, and the Cooperative Research Program of Network Joint Research Center for Materials and Devices, and Center for Spintronics Research Network (CSRN), Osaka University. Computations were performed at the Research Institute for Information Technology, Kyushu University.

\section{REFERENCES}

1. C. Li, A.J. Freeman, H.J.F. Jansen, and C.L. Fu, Phys. Rev. B 42, 5433 (1990).

2. K. Hotta, K. Nakamura, T. Akiyama, T. Ito, T. Oguchi, and A.J. Freeman, Phys. Rev. Lett. 110, 267206 (2013).

3. P. Bruno, Phys Rev. B 39, 865 (1989).

4. C. Andersson, B. Sanyal, O. Eriksson, L. Nordstöm, O. Karis, D. Arvanitis, T. Konishi, E. Holub-Krappe, and J. Hunter Dunn, Phys. Rev. Lett. 99, 177207 (2007).

5. J.P. Perdew, K. Burke, and M. Ernzerhof, Phys. Rev. Lett. 77, 3865 (1996).

6. E. Wimmer, H. Krakauer, M. Weinert, and A.J. Freeman, Phys. Rev. B 24, 864 (1981).

7. M. Weinert, E. Wimmer, and A.J. Freeman, Phys. Rev. B 26, 4571 (1982).

8. K. Nakamura, T. Ito, A.J. Freeman, L. Zhong, and J. Fernandez-de Castro, Phys. Rev. B 67, 014420 (2003).

9. R. Wu and A.J. Freeman, J. Magn. Magn. Mater. 200, 498 (1999).

10. M. Weinert, R.E. Watson, and J.W. Davenport, Phys. Rev. B 32,2115 (1985).

11. G.H.O. Daalderop, P.J. Kelly, and M.F.H. Schuurmans, Phys. Rev. B 41, 11919 (1990).

12. X.D. Wang, D.S. Wang, R.Q. Wu, and A.J. Freeman, J. Magn. Magn. Mater. 159, 337 (1996). 